\title{
HORMONAL IMPACT ON TUMOR GROWTH AND PROGRESSION
}

\author{
A. Reznikov \\ V.P. Komisarenko Institute of Endocrinology and Metabolism, the Academy of Medical Sciences \\ of Ukraine, 69 Vyshgorodskaya str., Kyiv 04114, Ukraine
}

In this review, current knowledge of hormones impact on prostate, breast and endometrium cancer growth and transition to hormone therapy resistant phenotype is summarized. A special consideration is given to genetic alterations and hormone responsive signaling pathways. Estrogen, androgen and progesterone are the key drivers in these cancer variants. Hormone receptors and their interplay with plenty of growth factors generated by the tumor microenvironment contribute greatly to carcinogenesis and cancer cell proliferation. The tumor cell plasticity that could explain a loss of responsiveness to hormone deprivation therapy and aggressive course of the diseases has been addressed.

Key Words: hormone, prostate cancer, breast cancer, endometrial cancer, receptor signaling.

\section{GENERAL ISSUES}

The hormones are known as all-round regulators of the cell growth, maturation and differentiation, as well function and metabolism. Their impact on tumor growth and progression can be direct or indirect. Some normal and malignant tissues demonstrate almost absolute dependence of growth upon steroid hormones. The issue of the initiating role of the androgens and estrogens in cancerous transformation of the prostatic, endometrial, breast and ovarian epithelium is still under discussion. In the meantime, there are no doubts of their direct promotional effect on these tumors growth and progression. An obvious example of indirect effect is an increased tumorigenic risk in chronic stress owing suppressive influence of adrenal corticosteroids on the immune system.

Coincidence of the response of normal and carcinoma's tissues to steroid hormones has created a basis of successful hormonal therapy of prostate, endometrium and breast cancers. As regards to the prostate cancer $(\mathrm{PCa})$, it was started with the remarkable milestone work by C. Huggins and C.V. Hodges [1]. In fact, this work was underlied by animal research discovery of postcastration atrophy of prostatic epithelium and impressive data on absence of $\mathrm{PCa}$ in the men who have been orchidectomized in childhood or adolescence.

All the three above-mentioned tumors emerge and develop like any others of different localization. However, it must be pointed that they mainly belong to hormone-dependent type, which determines their uniqueness and specificity.

Submitted: July 16, 2015

Correspondence: E-mail: reznikov39@gmail.com

Abbreviations used: $\mathrm{ADT}$ - androgen deprivation therapy;

$\mathrm{AR}$ - androgen receptor; $\mathrm{BCa}$ - breast cancer; DHT -

5a-dihydrotestosterone; ECa - endometrial cancer; ER - estrogen receptor; PCa - prostate cancer; PR - progesterone receptor; PSA prostate-specific antigen; SHBG - sex hormone binding globulin.
Steroid-dependent tumors cluster is characterized by a few common features as following:

1. The tumors are originated from epithelium layer, and most often identified by pathologist as carcinoma.

2. Hormones do not play essential role in initiation of carcinogenesis.

3. Deviations in hormone receptor and other signaling pathways seem to be of a great importance in initiating the tumor growth.

4. Because of mutations and chromosomal abnormalities, there are established links between steroid hormones and expression of oncogenic transcriptional factors. For this reason, steroids exert powerful effect on the cancer cells proliferation and promote the tumor progression.

5. BRCA1 seems to be involved into androgen and estrogen receptors signaling, so its mutations or deletion might apply to tumorigenesis and growth of prostate, breast and ovarian cancers.

6 . In the prostate, the mammary gland and endometrium, steroid hormones delegate many of their functions to local growth factors (EGF, FGF, NGF, VEGF, IGF-1, PDGF, and TGF). This indicates an importance of epithelial-stromal interrelationship in pathogenesis of hormone-dependent cancers.

7. Hormone deprivation therapy is successfully used for palliative treatment of prostate, breast and endometrium cancers and their metastasis.

8. Signaling rearrangements and changes in the rate of hormone-dependent and hormoneindependent cells inside tumor that occur during steroid hormone deprivation therapy usually lead to prevalence of hormone-independent cell type and therefore to resistance to this kind of treatment.

Whether colorectal cancer is a kind of estrogendependent tumor remains unclear.

\section{PROSTATE CANCER}

$\mathrm{PCa}$ is known as the most commonly diagnosed cancer and the second leading cause of death related to malignant tumors in male population of developed 
countries. In Ukraine, amount of PCa cases increased 2.4-fold since year 1991 till 2009, and the same rise was observed between 2005 and 2012. In 2013-2014, PCa was the third malignancy in the Ukrainian male population preceeded by respiratory organs and nonmelanoma skin cancers, and occupied the first place in the list of oncourology diseases [2].

In $80-85 \%$ of PCa (adenocarcinoma) cases, patients positively respond to androgen deprivation therapy (ADT). The crucial role of androgen steroids in PCa growth and progression is generally appreciated and confirmed by an increasing of experimental and clinical evidence. The tumor growth and dissemination can be retarded by orchidectomy or other ADT modalities including administration of androgen receptor (AR) antagonists, hypothalamic gonadoliberin ( $\mathrm{LH}-\mathrm{RH})$ agonists and antagonists, estrogens, and inhibitors of steroid 5a-reductase. These methods are aimed at suppression of testicular testosterone synthesis, metabolism or testicular and adrenocortical androgen activities.

In many PCa cases, efficacy of ADT is not such high as one expects. The attempts to find out correlation between blood serum testosterone levels and an incidence of PCa or the results of ADT failed. Nevertheless, many investigators have noticed an association of increased PCa risk and an increase in serum testosterone concentrations and a decrease in sex hormone binding globulin (SHBG) [3, 4], while the others were unable to confirm this $[5,6]$. According to collaborative analysis of 18 worldwide prospective studies that included 3886 men with incident PCa and 6438 control subjects serum concentrations of sex hormones (total testosterone, calculated free testosterone, $5 a$-dihydrotestosterone (DHT), dehydroepiandrosterone sulfate, androstenedione, androstanediol glucuronide, estradiol and calculated free estradiol) were not associated with the risk of PCa [4].

The results of our studies are in accordance with the reports on elevated testosterone in advanced and metastatic stages of the disease. There was a gain in SHBG levels in PCa patients with advanced disease when compared to those of healthy human males of comparable age [7] that corresponds to the data of other authors $[4,8]$.

Being mainly the "privilege" of aging men the risk of developing $\mathrm{PCa}$ might be associated with a low serum testosterone $(<8-12 \mathrm{nmol} / \mathrm{l})$. Before radical prostatectomy, there was found a nonlinear $\mathrm{U}$-shaped association of total preoperative testosterone and its correlation to estradiol with high-risk $\mathrm{PCa}$, so both the lowest and highest values of those parameters has achieved independent predictor status for a highrisk PCa [9]. Japanese authors reported an absence of a linkage between testosterone, SHBG levels and a risk of developing PCa [6]. However, a variant in $S H B G$ gene seems to implicate in prostate carcinogenesis, because SHBG D356N heterozygotes have about $34 \%$ higher risk of PCa among white population [10]. In prospective collaborative study, no clear impact of the serum total, free testosterone and other androgens as well as estrogens on PCa risk has been estimated [4].

Few studies supported an association between low testosterone in aging men and an increased risk of $\mathrm{PCa}$, high Gleason scores, aggressive behavior of the disease and worse survival [11-14]. What the aging hypogonadic men are more prone to develop PCa is not proved yet anyway. Should this hypothesis be true, a current knowledge on cellular and molecular mechanisms of prostatic carcinogenesis could unfold this paradox. At least, two explanations seem to be convincing enough to support this point of view as following:

1. Even low androgen concentrations are able to promote intraepithelial stem cell proliferation and, in some cases, an initial differentiation to oncogenic phenotype [15], perhaps due to fusion of oncogene encoding androgen-dependent transcription factor ERG to $5^{\prime}$-untranslated region of transmembrane protease serine (TMPRSS2) gene [16-18].

2. In the prostate physiology, testosterone and its active metabolite, DHT, directly stimulate a late terminal stage of acinar epithelium differentiation and secretory function. The same effect one can observe in cultured $\mathrm{LnCaP}$ cells which are widely used as androgen-sensitive PCa in vitro model, that is without growth factors produced by stromal elements. Presumably, in a case of low serum testosterone concentration, some transient or intermediate luminal epithelial cells generated from stem cells in adult prostate might remain undifferentiated and thereby involved in malignant transformation [15]. As a tumor starts to grow, it becomes sensitive to proliferative effect of testosterone and suppressive effect of ADT until transforming to ADT-resistant phenotype.

All those facts means that testosterone replacement therapy in aging men having low serum testosterone should promote terminal differentiation of prostatic epithelium without an increase of $\mathrm{PCa}$ risk. Indeed, clinical follow-up observations did not demonstrate such a complication despite appreciated fact on the presence of small sleeping tumors in the prostate of elderly men [19-21]. In general white population, these occult tumors are incidental findings by pathologist on post-mortal section in $12 \%$ of suddenly died organ donors starting from the $4^{\text {th }}$ decade of life [22].

Some PCa patients demonstrate aggressive tumor growth and progression, but other ones, especially elderly men, do not. There is a point of view, that PCa which affects an elderly man is a biological phenomenon of senescence [23]. The authors postulate that it depends on the proteins involved in control of regenerative potential, and late-life low-grade PCa which is characterized by indolent clinical course should be considered as a special PCa subcategory.

In summary, androgens could promote pre-existing PCa growth, but with long period of time before clinically manifested. There is no strong evidence of corre- 
lation between endogenous or exogenous androgens and $\mathrm{PCa}$ incidence, and further research is required.

Various chromosomal, mutational deviations and epigenetic modifications associated with $\mathrm{PCa}$ risk and the tumor have been described including mutations of CDKN1B, MCM7, IL11RA, HMGN1, the lost of heterozygosis at 8h22 locus, silencing of expression of TP53 gene, abnormal methylation in high-density C-G dinucleotide sequences regions of DNA, GSTP1, Laminin-5 genes and expression of CDKN2 gene which participates in the cell cycle control, and many others. CAG and GGC repeat length polymorphism in AR molecule is associated with a risk for PCa [24]. In the meantime, some authors could not confirm this for CAG repeats [5].

Multiple PCa risk variants were discovered on chromosome 8q24. In many advanced and metastatic $\mathrm{PCa}$ cases, changes of expression of RAS, MYC, BCL2 oncogenes and the genes encoding numerous growth factors were found. Also some other alterations occur such as hyperactivity of IKB5A1 and IKB5A2 genes encoding two isoenzimes of steroid 5-alpha-reductase which converts testosterone to DHT. It has been shown that hyperexpression of antiapoptotic gene TRPM2 commonly found in androgen-dependent tumor Shionogi is closely associated with its progression [25]. This gene is responsible for active cell death via apoptosis, and normally is repressed by androgens. Its overexpression leads to the loss of apoptotic potential. Recently a significant association between NUDT10 rs5945572 polymorphism and PCa risk was confirmed in a meta-analysis [26].

In accordance with a multifactorial hypothesis of prostate carcinogenesis [24], androgens augment the carcinogenic potention of endogenous or environmental carcinogens, such as some estrogen metabolites, reactive oxygen species, diet and genetic determinants.

It is commonly recognized that $\mathrm{AR}, \mathrm{P} 13 \mathrm{~K} / \mathrm{AKT}$ and PTEN signaling pathways are directly involved in $\mathrm{PCa}$ progression. In majority of primary PCa cases (approximately $80 \%$ ), androgens promote proliferation and inhibit apoptosis of the tumor epithelium cells like they do in normal prostate tissue. AR-mediated mechanism activates prostate-specific antigen (PSA) gene expression and, correspondingly, PSA production by acinar epithelium [27]. For many years the serum levels of total and free PSA are widely being used as a main biochemical markers for PCa diagnosis and control of its progression. Testosterone promotes angiogenesis due to enhancement of VEGF production. We observed angiogenesis in the prostate ventral lobe of castrated immature rats after testosterone replacement treatment [28].

It becomes evident from experimental studies in animal models that estrogens potentiate androgendependent PCa development and progression [29]. Estrogens originate from fat and presumably from PCa tissue through conversion of testosterone and androstendione with participation of the aromatase enzyme complex. Estrogen promoting effect is realized through estrogen receptor-alpha (ERa). High levels of this receptor type were found in hormone-refractory PCa as well as in the lymph nodes and metastasis.

The key role in androgen activities belongs to the AR signaling. That is why androgen ablation as surgical castration and combination treatment with $\mathrm{LH}-\mathrm{RH}$ agonists and $\mathrm{AR}$ antagonists which inhibit both testicular and adrenal androgens at the receptor level (maximum androgen blockade) are appreciated as effective palliative therapeutic modalities in terms of survival and quality of life in the patients with advanced or metastatic PCa. Alternatively, a low-dose estrogen-antiandrogen therapy can be used [7]

In the blood, testosterone is bound principally to SHBG and in less degree to albumin. About $2.5-3.0 \%$ of circulating testosterone is free and able to enter the cell, where it almost completely (about 90\%) irreversibly converted to DHT due to $5 a$-reductase activity. This enzyme is distributed in the prostate epithelium and stromal cells. Type $15 a$-reductase is localized mostly in the glandular epithelium, while type 2 prevails in the stroma.

According to F. Labrie's "intracrinology" concept, a significant portion of the human and primate prostate DHT originates from inactive or weak androgens (DEAS, androstenedione) produced by the adrenal cortex $[30,31]$. PCa is associated with empowered conversion of androstenedione to testosterone [32]. De novo synthesis of androgens in PCa tissue is capable of activating AR signaling program even in castrate-resistant stage of the tumor progression [33, 34].

The next intracellular events are similar for all steroid hormones. DHT binding to the prostatic cytoplasm AR that is coupled with heat-shock proteins is the first step of androgen signal transduction from receptor to effectors. Cytoplasm AR belongs to a superfamily of ligand-dependent transcriptional factors. DHT binding leads to dissociation of heat-shock proteins from the receptor, its phosphorilation, conformational modifications and dimerization. After translocation of the hormone-receptor complex into nucleus, DHT binds to the DNA androgen-responsive nucleotide sequences located within the promoters of androgenresponsive genes. In this case, DHT acts as a delivery tool for AR. This allows AR dimer in co-operation with other transcriptional factors and co-activators or co-repressors to regulate gene activity. Intratumoral testosterone which did not undergo 5 a reduction and represents a minor portion of prostatic androgens also is capable of binding AR though with less affinity than that of DHT. Hopefully, selective targeting the DNAbinding domain of AR represents a new approach to treatment of PCa [35].

Content of AR in PCa and its metastasis is extremely variable and obviously can not be used for prognosis and drug therapy selection. In many cases of lowdifferentiated PCa, it could not be found at all. In our study [7], no correlations between concentrations of cytoplasmic AR in the human PCa tissue and a stage 
of the disease or PSA levels were found out. This is not surprising because of heterogeneity of the tumor tissue composition. High variability of the epithelial and stromal ratio in PCa tissue samples that have been taken from the patients undergone radical prostatectomy contributes greatly to the results. It must be also taken into account that beside of a cytoplasmic AR there is its constitutive nuclear form, so some part of androgens can interact with it omitting cytoplasm receptor.

Intracellular AR signal pathways in PCa tissues dramatically change during the tumor progression [36-38]. PCa progression leads to androgenindependent state. Eventually PCa patients do not response to hormonal therapy (ADT) anymore. However, the tumor preserves ability to grow in presence of even extremely low levels of testosterone in its environment, for instance, after bilateral orchidectomy. Therefore, the terms "androgen-independent" or "androgenrefractory" does not mean absolute inability of $\mathrm{PCa}$ to be stimulated with androgen steroids of testicular or the adrenal cortex origin, it rather means resistance to hormonal therapy. Due to genetic and epigenetic adaptations, PCa remains sensitive to AR mediated growth signaling that support malignant cells surviving even after androgen ablation. However, high-grade advanced and metastatic PCa (G4) shows an attenuated androgen signaling protein and gene signature, based on study of 101 cell populations isolated with micro-dissection [39].

It must be taken into consideration that the tumor resistance to ADT can be caused by the membrane p-glycoprotein mediated drug efflux, and substitution of an antiandrogen for another one sometime resume therapeutic efficacy of androgen blockade.

The changes of expression of a number of AR coregulators (ARA 54, ARA 55, ARA 70, IL-6, ART 27, Her2/neu) were found in PCa cells and may influence on transcriptional activity of AR [40]. It has been shown that calcium ion signaling contributes to transition of LNCaP cells to androgen-insensitive, apoptosisresistant neuroendocrine phenotype [41, 42].

In a few PCa-derived cell lines, insulin promoted mitogenic activity directly through insulin receptor without participation of IGF-1 receptor [43]. In advanced stage of PCa growth, IGF-1 and other peptide growth factors (EGF, TGF- $\alpha$, TGF- $\beta$, FGFb) may activate the mutant $A R$ more efficiently then the wild-type receptor [44].

Amplification of AR gene in human PCa biopsies was described in $28 \%$ of patients who relapsed after endocrine therapy [45]. In all cases but one, the gene structure was normal. What is noticeable is that survival of patients with the gene amplification was significantly greater in comparison with those who did not demonstrate this phenomenon. Perhaps the longer AR signaling persists, the more possibility exists to support androgen-induced normal differentiation of the stem cells in the prostate.

To keep PCa cells in differentiated and ADTresponsible state as long as possible, the optimal androgen deprivation concept alternatively to maximal androgen blockade seems justified [46]. This approach includes combining therapeutics simultaneously targeting different mechanisms of PCa growth and progress, drug dosage reduction for decreasing cardiovascular and other complications, intermittent endocrine therapy with monitoring blood PSA levels, withdrawal treatment when remission was achieved, and replacement antiandrogen for another one in relapse. Similar strategy was recently proposed for treatment of castration-resistant PCa [47].

In animal research, we have studied an efficacy of combined drug treatments which interfere with different regulatory mechanisms of testosterone synthesis, metabolism and its action towards normal rat prostate and transplanted human neoplastic prostate tissues $[7,28,48-51]$. There were studied various dosage protocols and the combinations of nonsteroidal antiandrogen (flutamide), estrogens (diethylstilbestrol, hexestrol, chlorotrianizene, honvan), steroid $5 a$-reductase inhibitor (finasterid), LH releasing hormone (surfagon), recombinant cytokine EMAP II, etc. Pharmacodinamic effects were evaluated as characterized by axessory sexual glands morphology, DNA, RNA, protein and other ingredients synthesis and contents. Based on the results of the studies, a low dose estrogen-antiandrogen therapy has been implemented in clinical practice [7].

The role of AR amplification in pathogenesis and clinical course of PCa is still being discussed $[40,52$, 53]. Perhaps, this mechanism underlies capability of the tumor growth and progression to be stimulated by very low amount of androgens in the cells microenvironment.

PCa genome profiling shows a lot of different somatic mutations [54]. The authors suggest that they might determine so-called "endocrine-independent" PCa sensitivity to glucocorticoids, progestins and even to a new antiandrogen in "antiandrogen withdrawal syndrome". Glucocorticoid receptor pathway contributes to DHT-induced proliferation of castrationresistant PCa cells [55]. Though one nucleotide mutations of AR gene in PCa tissue are seldom found, they might contribute to the tumor progression and ADT refractoriness. Besides, the role of MATE transporters family has to be taken into account as one of the important mechanisms of drug resistance.

Cross-talk between AR and stromal growth factors signaling is of a key importance in normal prostate development and in pathogenesis of PCa [56]. It involves control of the cell differentiation, mitosis and programmed cell death (apoptosis). Androgen-growth factors interactions are disordered in androgen-independent PCa and cause sensitization of cancerous cells to low level of androgens in their microenvironment. It was shown that progesterone receptor (PR) signaling plays an active role in changing $\mathrm{PCa}$-associated stromal cell phenotype during the disease progression [57]. 
In non-malignant prostate and localized PCa, androgens stimulate acinar proliferation through stromaoriginated growth factors. Transition to androgenindependent state involves hyperexpression of stromal growth factors and unusual ability of cancerous epithelial cells to produce EGF, IGF-1, FGFb and some other peptides (the epithelium self-stimulation) as well as other ligand-independent mechanisms, such as cAMP, proteinkinases, and modulation of androgenmediated pathways including steroid biosynthesis and disregulated metabolism, amplification and mutation of $A R$ gene so on [58]. In other words, normal paracrine mechanism of prostate epithelium control switches to autocrine stimulation.

In a few PCa cell lines, insulin enchanced mitogenic activity directly via insulin receptor with no IGF-1 receptor to be involved [43]. In advanced PCa, IGF-1 and other growth factors (EGF, TGFa, TGF $\beta$, FGF $\beta$ ) may stimulate mutant $A R$ even more potent than normal AR [44].

An increasing number of evidence indicates that similarly to other malignant neoplasms there is a linkage between inflammation and PCa development and progression [59]. Chronic inflammation causes proliferative inflammatory atrophy. Among variety of the inflammation mediators, interleukines (IL)- 6 and IL-8 are of importance [40,60,61]. In two studied androgendependent PCa cell lines, LNCaP and 22Rv1, IL-8 drove cancerous cells to androgen-independent proliferation through expression and activation of AR. This might be one of the mechanisms of developing ADT refractory state because IL-8 promoted neuroendocine differentiation of PCa cells that is an early marker of transition to androgen-independent state.

It can not be excluded that in case of artificial androgen deprivation (ADT) androgen-dependent cells disappear through apoptosis, and are substituted with androgen-independent cell clones [62]. However, recent research results do not confirm this theory. Clonal progression of human PCa from Gleason grade 3 (G3) to grade 4 (G4) was studied in radical prostatectomy specimens using laser-captured microdissected tumors [63]. The TMPRSS:ERG gene fusion confirming common clonal origin has been found in all specimens studied. Some other genetic and epigenetic features proved that a subset of G3 (low-grade PCa) progress to G4 (advanced PCa) or they emerge from a common precursor. The TMPRSS:ERG gene fusion is a feature of aggressive androgen-independent $\mathrm{PCa}$, its expression can be regulated by a novel ER-dependent mechanism [64]. Whether PCa progression is rather a result of the tumor genetic and epigenetic adaptation than that of clonal selection is still not clear. It seems that ADT promotes the emergence of heterogeneous PCa cell populations that determine aggressive course of the disease [65].

\section{BREAST CANCER}

Breast cancer $(\mathrm{BCa})$ is acknowledged as a leading cause of death of women at the age of $40-55$ years.
It is soundly believed that an increased disease occurrence is facilitated by age-related endocrine rearrangements. Breast epithelium hyperplasia increases 4-9 times a risk of $\mathrm{BCa}$ and even more in a case of $\mathrm{BCa}$ family history. Risk factors include obesity (fat tissue produces estrogens and other hormones, as well as numerous growth factors and cytokines), ionizing radiation, environmental pollution with xenoestrogens, alcohol abuse, smoking, menopausal hormone replacement therapy, so on. High blood serum levels of estrone sulfate, prolactin and testosterone might be the predictors of BCa risk [66].

$\mathrm{BC}$ a originates from non-differentiated ductal epithelium ( $85 \%$ cases) or lobule type 1 epithelium (15\% cases), which prevail in nonparous women. In parous women, mammary gland epithelium is more differentiated (lobules type 3 or 4), therefore they are less predisposed to BCa development. Early menarche, infertility, late first pregnancy (after age of 35 years) increase a chance of the disease.

During puberty, ovarian cycles and pregnancy a large number of mammary stem cells undergoes differentiation in order to generate new tissue structures. This process is regulated by systemic hormones and locally produced paracrine peptides. According to the model proposed by C. Brisken and S. Duss [67], "repeated hormone stimulation of stem cells and their niches in the course of menstrual cycles may be an important early event in breast carcinogenesis and may explain the conundrum why $\mathrm{BCa}$ risk increases with the number of menstrual cycles experienced prior to a first pregnancy".

Numerous genetic damages were found in $\mathrm{BCa}$ cells and considered as markers of the disease: deletions of $8 p, 11 q, 13 q, 14 q$ chromosomal regions, elongation of $6 q 22,8 q 22,11 q 13,17 q 22-24$, $20 \mathrm{q} 13$, mutations of $B R C A 1$ and BRCA2 genes, and overexpression of ErbB2. Special attention should be given to aberrant variants of Foxp3 gene, which acts as suppressor of the oncogene HER-2/ErbB2. In a huge genome-wide association analysis covering about $120000 \mathrm{BCa}$ cases, SETBP1 at 18q12.3 and $R N F 115$ and $P D Z K 1$ at $1 \mathrm{q} 21.1$ were identified as main susceptibility loci for $\mathrm{BCa}$ [68].

$B C$ a susceptibility gene $B R C A 1$ is located on chromosome 17q21. It encodes an 1863 amino acid protein that is important for normal embryonic development, DNA repair, cell proliferation, inhibition of apoptosis, maintenance of the genome stability, steroid hormone receptor signaling and tumor growth suppression. BRCA2 protein performs the same functions. Both genes inactivating mutations are predictors of hereditary early onset BCa which occurs in 5-10\% of BCa cases and breast-ovarian cancer syndrome. Nearly half of women bearing these genes mutations develop BCa before age of $70[67,69]$.

Remarkable role of BRCA1 protein in pathogenesis of hormone-responsive cancers is determined by its linkage with sex steroid receptors. BRCA1 inhibits ERa activity and activates AR signaling $[70,71]$. 
It is considered that the local and extraovarian (in the fat tissue) biosynthesis of estrogens, especially in postmenopausal women, is of a great importance as pathophysiological mechanism in development of $\mathrm{BCa}$. Obesity is now associated with a low-grade inflammation, that via inflammation-induced cytokines increases steroid aromatase activity in the breast that results in a high local concentration of estrogens. Overexpression of CYP19 gene and increased aromatase and 17-beta-hydroxysteroid dehydrogenase activities are associated with more aggressive course of disease [72]. It seems that local catecholestrogens, hydroxylated products of estrogen metabolism, contributes to development of BCa.

Normal and malignant mammary epithelium grows and functions under multiple hormonal control. Numerous stromal paracrine and autocrine growth factors as IGF-1, TGFa, TGF $\beta$, EGA, FGF, PDGF and other mediate hormonal induction of mammary cells proliferation and differentiation, and in tumor progression $[73,74]$. In addition, adipocytes presenting in $\mathrm{BCa}$ microenvironment generate numerous endocrine, inflammatory and angiogenic substances that promote tumor growth. For instance, locally produced leptin induces androgen conversion to estrogens, which is important for BCa tumorigenesis [75].

For many years possible role of thyroid hormones as risk factors for $\mathrm{BCa}$ was being under discussion. Recently there were published the results of unique prospective study, in which 2185 women were observed during at average of 23.3 years. A positive correlation between pre-diagnosis blood plasma triiodothyronine levels and development of aggressive BCa, metastasis and the tumor negative ER and PR phenotypes has been revealed [76].

Since G.T. Beatson [77] had estimated the link between ovarian secretion and $\mathrm{BCa}$, majority of research was focused on the role of estrogens in its development and progression. Amongst plenty of other hormones, estrogens demonstrate the most apparent mitogenic potency. They play crucial role in normal breast development and carcinogenesis as permissive agents for carcinogens and tumor growth enhancers.

True role of estrogens in BCa growth and progress is controversial and not fully elucidated till now. They stimulate proliferation of ductal epithelial cells, but promote their differentiation and apoptosis, and interfere with metastatic dissemination due to an increase of cell adhesion. In the meantime, ovarian hormones promote malignant transformation of breast stem cells followed by disease reccurence and metastasis [78]

In the famous Women's Health Initiative study and similar research [79], menopausal women taking estrogen-progesterone replacement therapy had increased incidence of BCa during follow-up period. This was not a case of taking estrogen alone in the group of women with hysterectomies, and BCa incidence risk was even reduced. However, according to a number of prospective studies, there is the strongest correlation between higher total and free circulating estradiol levels and risk of BCa development in postmenopausal women, and some positive correlation was found in premenopausal subjects [80]. Surprisingly, in the European Prospective Investigation into Cancer and Nutrition with cohort of $801 \mathrm{BCa}$ cases and 1132 matched control subjects, the higher premenopausal blood plasma testosterone levels were strongly related to an increased BCa risk, although a possible morbidity increase was found with higher estradiol levels in BCa affected women under age 50 [81].

Biological effects of estrogens are mediated by two subtypes of estrogen receptors, ERa and ER $\beta$, which belong to superfamily of nuclear transcription factors. These isoforms mediate direct effect of estrogens on proliferation of epithelium and induction of synthesis of stromal growth factors. In particular, estrogens promote revascularization of the tumor by increasing VEGF production through transcriptional activation [82]. Recent research identified URB5, an ubiquitin ligase and known oncogene, as an agent targeting ERa levels and signaling, and modulating estrogen impact on BCa cells proliferation [83].

Likely to other steroid hormones, binding of estrogen to cytosolic ER leads to detachment of heat shock proteins, phosphorilation and dimerization of ER, followed by translocation to the nucleus. The dynamic structure of ER is studied in detail [84]. ER binds to estrogen responsive element of DNA as ligand-induced homodimer and regulates transcription in conjunction with co-activators, co-repressors and modulators. Transactivation domens, AF1 and AF2, are involved in regulation of transcriptional activity of ER. Carboxy terminal domain $\mathrm{F}$ modulates gene transcription.

In most cases of hormone-responsible BCa (about $75 \%$ ), ERa expression is present, whereas ER $\beta$ expression is decreased in comparison with normal tissue. There is a suggestion that ER $\beta$ exerts a differential effect on cell proliferation as compared with ERa and might counteract to proliferative ERa signaling in the tumor which is associated with tumor progression [85]. It must be noted, that only about $40 \%$ of $\mathrm{BCa}$ patients respond positively to antiestrogen therapy despite the presence of ER.

Along with $\mathrm{ER}, \mathrm{PR}$ expression in $\mathrm{BCa}$ tissue can be found in $65 \%$ of cases that is indicative of sensitivity to antiestrogen therapy, because PR synthesis depends on active ER. In $25 \%$ of patients, mostly those younger than 40 years, both ER and PR are not found that correlates with refractoriness to hormonal therapy, aggressive course of disease and poor prognosis.

Progesterone is involved in proliferation of the breast normal and malignant alveolar epithelium in the breast lobules. In these tissues, it stimulates luminal progenitor and stem cell population $[86,87]$. What is surprising is that mammary stem cells respond to steroid signaling, despite absence of the PR and ER [88]. Progesterone effects are mediated by two $\mathrm{PR}$ isoforms, $\mathrm{A}$ and $\mathrm{B}$, which are coded by one gene and normally present in approximately equal amount in luminal epithelium. In nondifferentiated $\mathrm{BCa}$, con- 
tent of the subtype A prevails, that usually can be found at early stage of the disease and is indicative of poor prognosis. Increased activity of the epidermal growth factor and methylation of $E R$ gene promoter is being found in a case of PR absence. Conversely, normal rate of subtypes $A$ and $B$ correlates with more differentiated tumor, and predicts latent course of the disease.

$\mathrm{PR}$ seems to play a dual role in regulation of $\mathrm{BCa}$ growth depending on PR isoforms signaling followed by stimulation or inhibition of cell proliferation [89]. In the breast basal-like PR-negative non-cancerous cells that have been exposed to ionizing radiation, progesterone generated cancer stem cells via membrane PR [90]. It was demonstrated recently that progesterone metabolite, 5a-dihydroprogesterone, stimulates mammary carcinogenesis, and this effect can be attenuated with steroid $5 a$-inhibitor, finasteride [91].

What is intriguing, AR expression in $\mathrm{BCa}$ tissue is usually found. However, the role of AR signaling in pathogenesis of $\mathrm{BCa}$ is not fully understood.

Aberrant cross-talk between steroid receptors and growth factors signaling is a key mechanism of $\mathrm{BCa}$ progression to hormone-independent growth. It makes the tumor non-responsible to antiestrogen treatment. Mutant ER is a frequent finding in hormone-resistant $\mathrm{BCa}$ [92]. Overexpression of epidermal growth factor receptor HER2/neu is of an importance. For example, resistance of $\mathrm{BCa}$ patients to tamoxifen, steroid ER modulator, which exhibits suppressive effect on growth of malignant breast epithelial cells due its antiestrogenic activity in this organ (but not in uterus), is associated with BCa tissue high levels of both HER2/ neu and ER [93]. Some cytoplasmic proteins, like vimentin and Notch, can be considered important indicators of BCa progression [94].

Recently a possible role of intracellular ferritin as a protein promoting growth of $\mathrm{BCa}$ has been demonstrated. In a few BCa cell lines, ferritin expression positively correlated with proliferative activity [95].

\section{ENDOMETRIAL CANCER}

The most common histopathological variant of endometrial cancer (ECa) is differentiated adenocarcinoma. ECa develops mainly in postmenopausal age with an average of 61 years. At this period of life the ovaries do not ovulate, and because of absence of postovulatory luteal bodies progesterone production decreases significantly and sometimes can not oppose effectively to proliferative influence of estrogens despite low estrogen levels. The most important risk factors are as following: atypical endometrial hyperplasia, early menarche, late menopause, high estrogen levels, overweight, nulliparity, family history, diabetes mellitus, estrogen monotherapy, hyperandrogenism, polycystic ovary syndrome, alcohol [96]. Hyperprolactinemia and hyperinsulinemia that often accompany polycystic ovary syndrome promote proliferation of normal and malignant endometrial surface and glandular epithelium. Moreover, expression of growth hormone, prolactin and corresponding mRNA in ECa tissues promotes tumor growth and indicates poor prognosis [97]. In endometrial tissue culture, insulin stimulates proliferation of epithelium and carcinogenesis. This effect is mediated by mitogen-activated proteinkinases and AKT signaling [98]. In 30-40\% patients, ECa develops despite postmenopausal endometrial atrophy, and typifies low differentiation.

It is well documented that high estradiol levels in postmenopausal women predict $2-4$ fold higher risk of ECa development as compared with low estradiol levels while premenopausal women do not demonstrate such association [99].

Mutations of PTEN and TP53, tumor suppression genes, were found in $83 \%$ and $20 \%$ of ECa cases, respectively [100]. Loss of PTEN protein expression in endometrial specimens is marked in ECa and atypical endometrial hyperplasia that precedes its emergence [101]. High expression of cyclooxygenase-2, which inhibits apoptosis and stimulates angiogenesis, is a common finding in well-differentiated ECa tissues. Low-differentiated cancers exhibit overexpression of p53 in conjunction with low expression of MDM2 and p14 ARF [102].

Endometrial epithelium develops and functions under multihormonal control; however, active estrogens are the main drivers of endometrial cell proliferation. Mitogen-activated protein kinases (MAPK) [103] and protein kinase $\mathrm{C} \alpha$ [104] mediate $17 \beta$-estradiolactivated ER signaling in the development and progression of ECa. Another mechanism of estrogenstimulated ECa cells growth is MAPK-mediated induction of telomerase activity [105].

It was postulated, that in nuclear ER-positive ECa cells proliferation is mediated by both ER-Notch and GPR 30-PI3K/AKT signaling, whereas in nuclear ER-negative ECa cells the first signaling pathway is not involved in cell proliferation [106].

Interestingly, epithelial cells proliferation is not arised in response to $17 \beta$-estradiol alone, they need to be stimulated with EGF, IGF-1 and other growth factors. It is widely recognized that significant increase in local blood flow and vascular permeability preceed estrogen-induced proliferation of endometrial epithelium. R.D. Koos [107] hypothesized that microvascular changes lead to penetration of IGF-I and other proteins into subepithelial stroma resulted in synergistic with ER-signaling proliferative effect. The vascular hyperpermeability is caused by estrogen-induced VEGF, this process involves such transcription factors as ERa and hypoxia-inducible factor 1 .

The shift of local interconversion between estrone and $17 \beta$-estradiol in favor of the last one in ECa grade 1 biopsies contributes to ERa-positive tumor growth [108]. Similarly to BCa, ECa growth can be stimulated by leptin produced by local adipocytes. It is noticeable that in co-cultivated endometrial fibroblasts and Ishikawa human ECa cell line leptin promotes carcinoma cell proliferation due to enhancement of aromatase activity followed by an increase of estradiol formation [109]. 
In addition to ERa and ERB, a novel G proteincoupled membrane ER, GPER/GPR30, was found to exert non-genomic effects of estrogens. High expression of GPER predicts poor ECa prognosis. In ERa-negative Hec50 ECa cells and xenografts, it mediates estrogen-activated ERK and PI3K via metalloproteinase activation followed by transactivation of EGFR that results in enhanced tumor growth [110].

Estrogen-dependent subtype accounts about $75 \%$ of ECa cases. Progesterone functions as an antagonist of estrogens, that is exerts antiproliferative effect due to several mechanisms. These mechanisms include a decrease of ER amount in tissues, stimulation of estrogen sulfotransferase and other estrogen-metabolizing enzymes, and targeting mitogenic signaling. For this reason high doses of progestins are in use for ECa treatment. Besides, progesterone promotes differentiation of endometrial cancerous cells followed by metaplasia, atrophy and apoptosis. Progesterone signaling starts from its binding to PR that is distributed in endometrial epithelium and stroma.

ER presence in ECa tissues was found in $30-50 \%$ of patients with prevalence of ERa subtype. PR-positive ECa (approximately $50 \%$ of patients) responds to progestin therapy, while advanced high grade tumor and hormone refractoriness are associated with amplification of HER2/neu gene and overexpression of consistent oncoprotein [111, 112].

\section{CONCLUDING REMARKS}

Despite substantial progress in understanding pathogenesis and advances in early detection and treatment, hormone-dependent cancers are still one of the leading causes of cancer death around the world. PCa, BCa and ECa might be considered as specific cluster of the malignant tumors. In many cases family history, blood hormone levels and cancerrelated genes predict a high risk of developing $\mathrm{PCa}$, $\mathrm{BCa}$ and $\mathrm{ECa}$ that could be rationale for intent observation of the corresponding individuals. As regards the risk of $\mathrm{PCa}$ development, its association with age-related low plasma testosterone level still needs to be clarified. Basic research of gonadal steroid receptors signaling and its cross-talk with growth factors and cytokines are promising for broadening current knowledge of mechanisms of hormone-dependent cancerogenesis and the tumor transition to hormonerefractory phenotype. Deeper insight into metabolic changes is of a big importance for that, as well as for comprehension of gonadal steroid receptors interplay with DNA, microRNA interference with gene expression and other key issues of development, growth and progress of hormone-dependent tumors. Further investigations are needed to clarify complicated molecular mechanisms of hormonal impact on tumor growth and progression in order to considerably improve outcomes of therapy.

\section{CONFLICT OF INTEREST}

The author declares that he has no conflict of interest.

\section{REFERENCES}

1. Huggins C, Hodges CV. Studies on prostatic cancer. I. The effect of castration, of estrogen and of androgen injection on serum phosphatases in metastatic carcinoma of the prostate. Cancer Res 1941; 1: 293-7.

2. Cancer in Ukraine 2013-2014. Incidence, mortality, activities of oncological service. Bull Natl Cancer Registry of Ukraine 2015; (16): 50-51.

3. Gann PH, Hennekens $\mathrm{CH}$, Ma J, et al. Prospective study of sex hormone levels and risk of prostate cancer. J Natl Cancer Inst 1996; 88: 1118-26.

4. Roddam AW, Allen NE, Appleby P, et al. Endogenous sex hormones and prostate cancer: a collaborative analysis of 18 prospective studies. J Natl Cancer Inst 2008; 100: 170-83.

5. Platz EA, Leitzmann MF, Rifai N, et al. Sex steroid hormones and the androgen receptor gene CAG repeat and subsequent risk of prostate cancer in the prostate-specific antigen era. Cancer Epidemiol Biomarkers Prev 2005; 14: 1262-9.

6. Sawada N, Iwasaki M, Inoue M, et al. Plasma testosterone and sex hormone-binding globulin concentrations and the risk of prostate cancer among Japanese men: A nested case-control study. Cancer Sci 2010; 101: 2652-7.

7. Vozianov A, Reznikov A, Klimenko I. Androgen deprivation strategy in prostate cancer. Kyiv: Naukova Dumka. Ternopil: Ukrmedknyga, 2001. 240 p.

8. Schnoeller T, Jentzmik F, Rinnab L, et al. Circulating free testosterone is an independent predictor of advanced disease in patients with clinically localized prostate cancer. World J Urol 2013; 31: 253-9.

9. Salonia A, Abdollah F, Capitanio U, et al. Serum sex steroids depict a nonlinear U-shaped association with highrisk prostate cancer at radical prostatectomy. Clin Cancer Res 2012; 18: 3648-57.

10. Berndt SI, Chatterjee N, Huang W-Y, et al. Variant in sex hormone-binding globulin gene and the risk of prostate cancer. Cancer Epidemiol Biomarkers Prev 2007; 16: 165-8.

11. Gann PH, Hennekens $\mathrm{CH}$, Ma J, et al. Prospective study of sex hormone levels and risk of prostate cancer. $\mathbf{J}$ Natl Cancer Inst 1996; 88: 1118-26.

12. Morgentaler A. Goodbye androgen hypothesis, hello saturation model. European Urol 2012; 62: 765-7.

13. Isom-Batz G, Bianco FJ Jr, Kattan MW, et al. Testosterone as a predictor of pathological stage in clinically localized prostate cancer. J Urol 2005; 173: 1935-7.

14. Marks LS, Mazer NA, Mostaghel E, et al. Effect of testosterone replacement therapy on prostate tissue in men with late-onset hypogonadism: a randomized controlled trial. JAMA 2006; 296: 2351-61.

15. Van der Griend DJ, d'Antonio JM, Isaacs JT. Hormonal regulation of the prostate. In: Berges R, Tombal B, eds. Androgens and prostate cancer. Ismar Healthcare Publ 2008: 11-33.

16. Soller MJ, Isaksson $M$, Elfving $P$, et al. Confirmation of the high frequency of the TMPRSS2/ERG fusion gene in prostate cancer. Genes Chromosomes Cancer 2006; 45: 717-9.

17. Hermans KG, van Marion $R$, van Dekken $H$, et al. TMPRSS2:ERG fusion by translocation or interstitial deletion is highly relevant in androgen-dependent prostate cancer, but is bypassed in late-stage androgen receptor-negative prostate cancer. Cancer Res 2006; 66: 10658-63.

18. Clark J, Merson S, Jhavar S, et al. Diversity of TMPRSS2-ERG fusion transcripts in the human prostate. Oncogene 2007; 26: 2667-73.

19. Kaplan AL, Hu JC. Use of testosterone replacement therapy in the United States and its effect on subsequent prostate cancer outcomes. Urology 2013; 82: 321-6. 
20. Morgentaler A. Testosterone therapy in men with prostate cancer: scientific and ethical considerations. J Urol 2013; 189: S26-33.

21. Baillargeon J, Kuo YF, Fang X, Shahinian VB. Longterm exposure to testosterone therapy and the risk of high-grade prostate cancer. J Urol 2015; pii: S0022-5347(15)04148-8. doi: 10.1016/j.juro.2015.05.099. [Epub ahead of print].

22. Yin M, Bastacky S, Chandran U, et al. Prevalence of incidental prostate cancer in the general population: a study of healthy organ donors. J Urol 2008; 179: 892-5.

23. Drewa T, Jusinski M, Marzalek A. Prostate cancer which affects an elderly man is a feature of senescence (cellular) - a biology phenomenon. Exp Oncol 2010; 32: 228-32.

24. Bosland MC. Chapter 2: The role of steroid hormones in prostate carcinogenesis. J Natl Cancer Inst Monogr 2000; 27: $39-66$

25. Rennie PS, Bruchovsky N, Akakura K, et al. Apoptosis in relation to androgen independence in experimental and clinical prostate cancer. In: Tenniswood M, Michna H, eds. Apoptosis in hormone-dependent cancers. Springer-Verlag Berlin Heidelberg, 1995: 71-82.

26. Wencheng L, Min G. NUDT11 rs5945572 polymorphism and prostate cancer risk: a meta-analysis. Int J Clin Exp Med 2015; 8: 3474-81.

27. Kim J, Coetzee GA. Prostate specific antigen gene regulation by androgen receptor. J Cell Biochem 2004; 93: $233-41$.

28. Reznikov A, Chaykovskaya L, Polyakova L, Sachynska O. Effects of cytokine-like polypeptide EMAP II and flutamide on testosterone-stimulated prostate of castrated rats. Int J Physiol Pathophysiol 2012; 3: 161-73.

29. Bonknoff $\mathrm{H}$, Berges $\mathrm{R}$. The role of oestrogens in prostate cancer. In: Berges R, Tombal B, eds. Androgens and prostate cancer. Ismar Healthcare Publ, 2009: 49-59. Labrie F, Belanger A, Dupont A, et al. Science behind total androgen blockade: from gene to combination therapy. Clin Invest Med 1993; 16: 475-92.

30. Labrie F, Belanger A, Dupont A, et al. Science behind total androgen blockade: from gene to combination therapy. Clin Invest Med 1993; 16: 475-92.

31. Labrie F, Dupont A, Cusan L. Major advantages of "early" administration of endocrine combination therapy in advanced prostate cancer. Clin Invest Med 1993; 16: 493-8.

32. Gianfrilli D, Pierotti S, Pofi R, et al. Sex steroid metabolism in benign and malignant intact prostate biopsies: individual profiling of prostate intracrinology. Biomed Res Int 2014; 2014: 464869. doi: 10.1155/2014/464869.

33. Cai $\mathrm{C}$, Chen $\mathrm{S}, \mathrm{Ng} \mathrm{P}$, et al. Intratumoral de novo steroid synthesis activates androgen receptor in castrationresistant prostate cancer and is upregulated by treatment with CYP 17A1 inhibitors. Cancer Res 2011; 71: 6503-13.

34. Locke JA, Guns ES, Lubik AA, et al. Androgen levels increase by intratumoral de novo steroidogenesis during progression of castration-resistant prostate cancer. Cancer Res 2008; 68: 6407-15

35. Dalal K, Roshan-Moniri M, Sharma A, et al. Selectively targeting the DNA-binding domain of the androgen receptor as a prospective therapy for prostate cancer. J Biol Chem 2014; 289: 26417-29.

36. Sharifi N, McPhaul MJ, Auchus RJ. "Getting from here to there" - mechanisms and limitations to the activation of the androgen receptor in castration-resistant prostate cancer. J Investig Med 2010; 58: 938-44.

37. Bluemn EG, Nelson PS. The androgen/androgen receptor axis in prostate cancer. Curr Opin Oncol 2012; 24: $251-7$
38. Egan A, Dong Y, Zhang H, et al. Castration-resistant prostate cancer: adaptive responses in the androgen axis. Cancer Treat Rev 2014; 40: 426-33.

39. Tomlins SA, Mehra R, Rhodes DR, et al. Integrative molecular concept modeling of prostate cancer progression. Nature Genetics 2006; 39: 41-51.

40. Golias Ch, Iliadis I, Peschos D, Charalabopoulos K. Amplification and co-regulators of androgen receptor gene in prostate cancer. Exp Oncol 2009; 31: 3-8.

41. Vanden Abeele F, Shuba Y, Roudbaraki M, et al. Store-operated $\mathrm{Ca}^{2+}$ channels in prostate cancer epithelial cells: function, regulation, and role in carcinogenesis. Cell Calcium 2003; 33: 357-73.

42. Prevarskaya N, Skryma R, Shuba Ya, et al. $\mathrm{Ca}^{2+}$ homeostasis in apoptotic resistance of prostate cancer cells. Biochem Biophys Res Com 2004; 322: 1326-35.

43. Weinstein D, Sarfstein R, Laron Z, Werner H. Insulin receptor compensates for IGF1R inhibition and directly induces mitogenic activity in prostate cancer cells. Endocr Connect 2014; 3: 24-35.

44. Ruijter E, van de Kaa Ch, Miller G, et al. Molecular genetics and epidemiology of prostate carcinoma. Endocr Rev 1999; 20: 22-45.

45. Koivisto P, Kononen J, Palmberg C, et al. Androgen receptor gene amplification: a possible molecular mechanism for androgen deprivation therapy failure in prostate cancer. Cancer Res 1997; 57: 314-9.

46. Reznikov AG. Optimum androgen blockade - a novel concept in endocrine therapy of prostate cancer patients. Oncology 1999; 1: 85-9 (in Ukrainian).

47. Mitsiades N. A road map to comprehensive androgen receptor axis targeting for castration-resistant prostate cancer. Cancer Res 2013; 73: 4599-605.

48. Chaykovskaya LV, Polyakova LI, Sachynska OV, Reznikov AG. Potentiation of antiandrogenic effects of flutamide by synestrol low doses. Klin Exp Patol 2006; (2): 2-9 (in Ukrainian).

49. Reznikov AG, Chaykovskaya LV, Polyakova LI, Grigorenko VN. Effect of dexamethazone, flutamide and their combination on the xenografts of human prostate adenocarcinoma in mouse model. J AMN of Ukrainy 2009; (4): 802-9 (in Ukrainian).

50. Reznikov AG, Chaykovskaya LV, Polyakova LI, et al. Cooperative antitumor effect of endothelial-monocyre activating polypeptide II and flutamide on human prostate cancer xenografts. Exp Oncol 2011; 33: 231-4.

51. Polyakova LI, Salivonyk OA, Chaykovskaya LV, et al. Experimental study of antitumor effect of a new hormone combination in xenograft model of human prostate cancer. Liky Ukrainy 2012; (10 (166)): 86-9 (in Ukrainian).

52. Linja MJ, Visakorpi T. Alterations of androgen receptor in prostate cancer. J Steroid Biochem Mol Biol 2004; 92: $255-64$.

53. Palmberg C, Koivisto $P$, Kakkola L, et al. Androgen receptor gene amplification at primary progression predicts response to combined androgen blockade as second line therapy for advanced prostate cancer. J Urol 2000; 164: 1996-7.

54. Golias Ch, Charalabopoulos A, Stagikas D, et al. Molecular profiling and genomic microarrays in prostate cancer. Exp Oncol 2007; 29: 82-4.

55. Song C, Kim Y, Min GE, Ahn H. Dihydrotestosterone enhances castration-resistant prostate cancer cell proliferation through STAT5 activation via glucocorticoid receptor pathway. Prostate 2014; 74: 1240-8.

56. Zhu ML, Kyprianou N. Androgen receptor and growth factor signaling cross-talk in prostate cancer cells. Endocr Relat Cancer 2008; 84: 841-9. 
57. Yu Y, Yang O, Fazli L, et al. Progesterone receptor expression during prostate cancer progression suggests a role of this receptor in stromal cell differentiation. Prostate 2015; 75: $1043-50$.

58. Schalken JA. Molecular aspects of hormone-independent prostate cancer. In: Berges R, Tombal B, eds. Androgens and prostate cancer. Ismar Healthcare Publ, 2009: 37-46.

59. De Marzo AM, PlatzEA, Sutcliffe $S$, et al. Inflammation in prostate carcinogenesis. Nat Rev Cancer 2007; 7: 256-69.

60. Deeble PD, Murphy DJ, Parsons SJ, Cox ME. Interleukin-6- and cyclic AMP-mediated signaling potentiates neuroendocrine differentiation of $\mathrm{LNCaP}$ prostate tumor cells. Mol Cell Biol 2001; 21: 8471-82.

61. Seaton A, Scullin P, Maxwell PJ, et al. Interleukin-8 signaling promotes androgen-independent proliferation of prostate cancer cells via induction of androgen receptor expression and activation. Carcinogenesis 2008; 29: 1148-56.

62. Oldridge EE, Pellacani D, Collins AT, Maitland NJ. Prostate cancer stem cells: are they androgenresponsive? Mol Cell Endocrinol 2012; 360: 14-24.

63. Sowalsky AG, Ye H, Bubley GJ, Balk SP. Clonal progression of prostate cancers from Gleason grade 3 to grade 4 . Cancer Res 2013; 73: 1050-5.

64. Setlur SR, Mertz KD, Hoshida Y, et al. Estrogendependent signaling in a molecularly distinct subclass of aggressive prostate cancer. J Natl Cancer Inst 2008; 100: 815-25.

65. Bishop JL, Davies A, Ketola K, Zoubeidi A. Regulation of tumor cell plasticity by the androgen receptor in prostate cancer. Endocr Relat Cancer 2015; 22: R165-82.

66. Tworoger SS, Zhang X, Eliassen $\mathrm{AH}$, et al. Inclusion of endogenous hormone levels in risk prediction models of postmenopausal breast cancer. J Clin Oncol 2014; 32: 3111-7.

67. Brisken C, Duss S. Stem cells and the stem cell niche in the breast: an integrated hormonal and developmental perspective. Stem Cell Rev 2007; 3: 147-56.

68. Michailidou K, Beesley J, Lindstrom S, et al. Genomewide association analysis of more than 120,000 individuals identifies 15 new susceptibility loci for breast cancer. Nat Genet 2015; 47: 373-80.

69. Chen S, Parmigiani G. Meta-analysis of BRCA1 and BRCA2 penetrance. J Clin Oncol 2007; 25: 1329-33.

70. Rosen EM, Fan S, Pestell RG, Goldberg ID. BRCA1 in hormone-responsive cancers. Trends Endocrinol Metab 2003; 14: 378-85.

71. Rosen EM, Fan S, Isaacs C. BRCA1 in hormonal carcinogenesis: basic and clinical research. Endocr Relat Cancer 2005; 12: 533-48.

72. Subramanian A, Salhab M, Mokbel K. Oestrogen producing enzymes and mammary carcinogenesis: a review. Breast Cancer Res Treat 2008; 111: 191-202.

73. Lippman ME, Dickson RB. Mitogenic regulation of normal and malignant breast epithelium. Yale J Biol Med 1989; 62: 459-80.

74. Hawsawi Y, El-Gendy R, Twelves C, et al. Insulin-like growth factor - oestradiol crosstalk and mammary gland tumourigenesis. Biochim Biophys Acta 2013; 1836: 345-53.

75. Liu L, Wang L, Zheng J, Tang G. Leptin promotes human endometrial carcinoma cell proliferation by enhancing aromatase (P450arom) expression and estradiol formation. Eur J Obstet Gynecol Reprod Biol 2013; 170: 198-201.

76. Tosovic A, Bondeson AG, Bondeson L, et al. T3 levels in relation to prognostic factors in breast cancer: a populationbased prospective cohort study. BMC Cancer 2014; 14: 536-45.

77. Beatson GT. On the treatment of inoperable cases of carcinoma of the mamma: suggestions for a new method of treatment with illustrative cases. Lancet 1896; 148/2: 162-5.
78. Nandy SB, Gangwani L, Nahleh Z. Recurrence and metastasis of breast cancer is influenced by ovarian hormone's effect on breast cancer stem cells. Future Oncol 2015; 11: 983-95.

79. Chlebowski RT, Manson JE, Anderson GL, et al. Estrogen plus progestin and breast cancer incidence and mortality in the Women's Health Initiative Observational Study. J Natl Cancer Inst 2013; 105: 526-35.

80. Folkerd E, Dowsett M. Sex hormones and breast cancer risk and prognosis. Breast 2013; (Suppl 2): S38-43.

81. Kaaks R, Tikk K, Sookthai D, et al. Premenopausal serum sex hormone levels in relation to breast cancer risk, overall and by hormone receptor status - results from the EPIC cohort. Int J Cancer 2014; 134: 1947-57.

82. Applanat MP, Buteau-Lozano H, Herve MA, Corpet A. Vascular endothelial growth factor is a target gene for estrogen receptor and contributes to breast cancer progression. Adv Exp Med Biol 2008; 617: 437-44.

83. Bolt MJ, Stossi F, Callison AM, et al. Systems levelbased RNAi screening by high content analysis identifies UBR5 as a regulator of estrogen receptor- $\alpha$ protein levels and activity. Oncogene 2015; 34: 154-64.

84. Kumar R, Zakharov MN, Khan SH, et al. The dynamic structure of the estrogen receptor. J Amino Acids 2011; 2011: ID 812540. http://dx.doi.org/10.4061/2011/812540.

85. Omoto $Y$, Iwase $H$. Clinical significance of estrogen receptor $\beta$ in breast and prostate cancer from biological aspects. Cancer Sci 2015; 106: 337-43.

86. Axlund SD, Sartorius CA. Progesterone regulation of stem and progenitor cells in normal and malignant breast. Mol Cell Endocrinol 2012; 357: 71-9.

87. Hilton HN, Santucci N, Silvestri A, et al. Progesterone stimulates progenitor cells in normal human breast and breast cancer cells. Breast Cancer Res Treat 2014; 143: 423-33.

88. Asselin-Labat ML, Vaillant F, Sheridan JM, et al. Control of mammary stem cell function by steroid hormone signalling. Nature 2010; 465: 798-802.

89. Giulianelli S, Molinolo A, Lanari C. Targeting progesterone receptors in breast cancer. Vitam Horm 2013; 93: 161-84.

90. Vares G, Sai S, Wang B, et al. Progesterone generates cancer stem cells through membrane progesterone receptortriggered signaling in basal-like human mammary cells. Cancer Lett 2015; 362: 167-73.

91. Wiebe JP, Rivas MA, Mercogliano MF. Progesterone-induced stimulation of mammary tumorigenesis is due to the progesterone metabolite, $5 \alpha$-dihydroprogesterone $(5 \alpha \mathrm{P})$ and can be suppressed by the $5 \alpha$-reductase inhibitor, finasteride. J Steroid Biochem Mol Biol 2015; 149: 27-34.

92. Toy W, Shen Y, Won H, et al. ESR1 ligand-binding domain mutations in hormone-resistant breast cancer. Nat Genet 2013; 45: 1439-45.

93. Shou J, Massarweh S, Osborne CK, et al. Mechanisms of tamoxifen resistance: increased estrogen receptor-HER2/ neu cross-talk in ER/HER2-positive breast cancer. J Natl Cancer Inst 2004; 96: 926-35.

94. Calaf GM, Balajee AS, Montalvo-Villagra MT, et al. Vimentin and Notch as biomarkers for breast cancer progression. Oncol Lett 2014; 7: 721-7.

95. Chekhun SV, Lukyanova NY, Shvets YV, et al. Significance of ferritin expression in formation of malignant phenotype of human breast cancer cells. Exp Oncol 2014; 36: 179-83.

96. Tinelli A, Vergara D, Martignago R, et al. Hormonal carcinogenesis and socio-biological development factors in endometrial cancer: a clinical review. Acta Obstet Gynecol Scand 2008; 87: 1101-13. 
97. Wu ZS, Yang K, Wan Y, et al. Tumor expression of human growth hormone and human prolactin predict a worse survival outcome in patients with mammary or endometrial carcinoma. J Clin Endocrinol Metab 2011; 96: E1619-29.

98. Bishop EA, Lightfoot $\mathrm{S}$, Thavathiru E, Benbrook DM. Insulin exerts direct effects on carcinogenic transformation of human endometrial organotypic cultures. Cancer Invest 2014; 32: 63-70.

99. Brown SB, Hankinson SE. Endogenous estrogens and the risk of breast, endometrial, and ovarian cancers. Steroids 2015; 99: 8-10.

100. Boruban MC, Altundag K, Kilic GS, Blankstein J. From endometrial hyperplasia to endometrial cancer: insight into the biology and possible medical preventive measures. Eur J Cancer Prev 2008; 17: 133-8.

101. Lacey JV, Mutter GL, Ronnett BM, et al. PTEN expression in endometrial biopsies as a marker of progression to endometrial carcinoma. Cancer Res 2008; 68: 6014-20.

102. Buchynska LG, Nesina IP, Kashuba EV. Different trends of p53, MDM2 and p14 ${ }^{\mathrm{ARF}}$ expression patterns in endometrial adenocarcinomas versus hyperplasia. Exp Oncol 2007; 29: 287-94.

103. Zhou L, Cai B, Bao W, et al. Crosstalk between estrogen receptor and mitogen-activated protein kinase signaling in the development and progression of endometrial cancer. Int J Gynecol Cancer 2011; 21: 1357-65.

104. Thorne AM, Jackson TA, Willis VC, Bradford AP. Protein kinase C $\alpha$ modulates estrogen-receptor-dependent transcription and proliferation in endometrial cancer cells. Obstet Gynecol Int 2013; 2013: 537479. doi: 10.1155/2013/537479.
105. Zhou C, Steplowski TA, Dickens HK, et al. Estrogen induction of telomerase activity through regulation of the mitogen-activated protein kinase (MAPK) dependent pathway in human endometrial cancer cells. PLoS One 2013; 8: e55730.

106. Wei $Y$, Zhang Z, Liao H, et al. Nuclear estrogen receptor-mediated Notch signaling and GPR30-mediated PI3K/AKT signaling in the regulation of endometrial cancer cell proliferation. Oncol Rep 2012; 27: 504-10.

107. Koos RD. Minireview: Putting physiology back into estrogens' mechanism of action. Endocrinology 2011; 152: $4481-8$.

108. Cornel KM, Kruitwagen RF, Delvoux B, et al. Overexpression of $17 \beta$-hydroxysteroid dehydrogenase type 1 increases the exposure of endometrial cancer to $17 \beta$-estradiol. J Clin Endocrinol Metab 2012; 97: E591-601.

109. Liu L, Wang L, Zheng J, Tang G. Leptin promotes human endometrial carcinoma cell proliferation by enhancing aromatase (P450arom) expression and estradiol formation. Eur J Obstet Gynecol Reprod Biol 2013; 170: 198-201.

110. Petrie WK, Dennis $\mathrm{MK}, \mathrm{Hu} \mathrm{C}$, et al. G proteincoupled estrogen receptor-selective ligands modulate endometrial tumor growth. Obstet Gynecol Int 2013; 2013: 472720. doi: 10.1155/2013/472720. Epub 2013 Nov 27.

111. Bainazarova AA, Iskakova ZhK. Correlation between receptor status and results of treatment in endometrial carcinoma. Vopr Onkol 2006; 52: 654-8 (in Russian).

112. Grushko TA, Filiaci VL, Mundt AJ, et al. An exploratory analysis of HER-2 amplification and overexpression in advanced endometrial carcinoma: a Gynecologic Oncology Group study. Gynecol Oncol 2008; 108: 3-9. 